\title{
The very local Hubble flow: simulating the transition from chaos to order
}

\author{
A. D. Chernin ${ }^{1,2}$, I. D. Karachentsev ${ }^{3}$, M. J. Valtonen ${ }^{1,4}$, V. P. Dolgachev ${ }^{2}$, L. M. Domozhilova ${ }^{2}$, and D. I. Makarov ${ }^{3,5}$ \\ 1 Tuorla Observatory, Turku University, Piikkiö, 21 500, Finland \\ 2 Sternberg Astronomical Institute, Moscow University, Moscow, 119899, Russia \\ 3 Special Astrophysical Observatory, Nizhnii Arkhys, 369167, Russia \\ ${ }^{4}$ University of West Indies, Trinidad and Tobago \\ 5 Isaac Newton Institute of Chile, SAO Branch, Russia
}

Received 17 May 2005 / Accepted 29 January 2007

\section{ABSTRACT}

\begin{abstract}
The physical nature of the very local $(<3 \mathrm{Mpc})$ Hubble flow is studied on the basis of the recent high-precision observations in the Local Volume. A model including both analytical treatment and computer simulations describes the flow's dynamical evolution from a chaotic Little Bang initial state to the present-day state of a quasi-regular expansion. The dynamical effect of the uniform cosmic vacuum (time-independent dark energy or the cosmological constant) is taken into account.
\end{abstract}

Key words. galaxies: Local Group

\section{Introduction}

It has long been taken for granted in theoretical cosmology that the notion of the cosmological expansion is applicable to only very large spatial distances and that only at the scale of great clusters of galaxies, the markers participating in the cosmological expansion, can be found. A reason for this comes from the expansion with the linear velocity-distance relation being directly associated with the uniformity of the Universe in the Friedmann standard cosmological theory. Since the matter distribution is only uniform on spatial scales larger than 100-300 Mpc, the cosmological expansion is treated as a phenomenon of the largest observed scales. However, in drastic contradiction with this view, the phenomenon was originally discovered by Hubble in the local volume within $20 \mathrm{Mpc}$ from us. This volume is deep inside the cosmic cell of matter uniformity, and observations reveal a significant non-uniformity in the spatial distribution of galaxies there. How can the regular linear velocity field be compatible with the observed spatial non-uniformity of the galaxy distribution in the local volume? How might the cosmological phenomenon be discovered so close to us?

The questions have been stated clearly by Sandage (1986; see also Sandage et al. 1972). In a recent paper, Sandage (1999) concludes that an "explanation of why the local expansion field is so noiseless remains a mystery". It is also puzzling that the local rate of expansion is similar to the global one, if not exactly the same, with 10-15 percent accuracy (Sandage 1999). This is the Hubble-Sandage paradox described in more detail in our earlier paper (Chernin et al. 2004 - hereafter Paper I).

A possible solution to the Hubble-Sandage paradox has been suggested by Chernin et al. (2000) soon after the discovery of cosmic vacuum (dark energy or the cosmological constant) in distant Ia supernova observations (Riess et al. 1998; Perlmutter et al. 1999). It has been recognized (Chernin 2001; Baryshev et al. 2001) that the cosmic vacuum, with its perfectly uniform density, makes the Universe effectively uniform on various spatial scales, both large and relatively small, where cosmic vacuum dominates by density over dark matter and baryons. The dynamical effect of the cosmic vacuum is enhanced by the effective gravitating density of vacuum being $\rho_{\mathrm{V}}+3 p_{\mathrm{V}}=-2 \rho_{\mathrm{V}}$, where $p_{\mathrm{V}}=-\rho_{\mathrm{V}}$ is the vacuum pressure. In the Local Volume, vacuum dominates by force at distances greater than $R_{\mathrm{V}} \sim 1 \mathrm{Mpc}$ from the barycenter of the Local Group. On the "zero-gravity surface" of the size $\simeq R_{\mathrm{V}}$, the gravity of the Local Group dark matter and baryons is balanced by the antigravity of vacuum. Observations show that these are just the distances from which the observed Hubble flow starts.

These considerations suggest that the cosmic vacuum may control the dynamics of the observed Universe on both global spatial scales approaching the observation horizon and local scales deep inside the cell of matter uniformity. Because of this, the cosmological expansion may be not only a global phenomenon, but also a local one. On all those spatial scales where vacuum dominates, the expansion rate may be exactly or nearly the same, since the rate (measured by the Hubble factor) is mainly determined by one and the same physical agent that is a vacuum with its perfectly uniform density (Karachentsev et al. 2003a). In other words, “...the total force field is nearly homogeneous (smooth) due to the dominance of an all-pervasive cosmological constant, diluting any lumpy gravity field of the clustered matter..." (Thim et al. 2003).

The part of the expansion flow that is nearest to us, the very local ( $<3 \mathrm{Mpc}$ ) Hubble flow (VLHF), is obviously of special interest as the area where the expansion originates. In Paper I, we presented the most recent data on the VLHF kinematics, including original distance measurements made mostly with the Hubble Space Telescope (Karachentsev et al. 2000, 2002a, 2002b, 2003b; see also Karachentsev 2005; Karachetsev et al. 2006). The data display the VLHF as a well-organized outflow with the linear velocity-distance relation. Its expansion rate is $72 \pm 7 \mathrm{~km} \mathrm{~s}^{-1} \mathrm{Mpc}^{-1}$ and the one-dimension, random mean 
velocity is about $20 \mathrm{~km} \mathrm{~s}^{-1}$. As a first step in the search for the physical nature of the VLHF, we performed a set of computer simulations to reconstruct the dynamical history of the flow. We found (Paper I) that the evolution of the VLHF might begin at the epoch of the Local Group formation some 12-13 Gyr ago. At that time, the VLHF galaxies, together with the forming major galaxies of the group and a variety of subgalactic units, participated in violent nonlinear dynamics with multiple collisions and merging.

This violent initial state is described by the Little Bang model (Byrd et al. 1994), and it also agrees with the concept of the early Local Group (van den Bergh 2003). We assumed that the VLHF might be formed by relatively small units that have survived accretion by the major galaxies and managed to escape from the gravitational potential well of the Local Group to the area outside the local zero-gravity surface. A typical VLHF member galaxy gains escape velocity from the non-stationary gravity field of the forming group and a velocity higher than some $100-150 \mathrm{~km} \mathrm{~s}^{-1}$ enables it to reach the vacuum-dominated area where it gains additional acceleration due to the antigravity of the cosmic vacuum.

According to the results of Paper I, the VLHF is not a slightly disturbed initial cosmological flow where the linear velocity law existed from the "very beginning". On the contrary, the observed members of the VLHF underwent strong nonlinear interactions with the major galaxies of the Local Group in the group volume before they formed the quasi-regular flow outside the zero-gravity surface. Their initially chaotic motions became quasi-regular under the combined action of the quasi-static and quasi-spherically symmetrical gravitational potential of the Local Group outside the local zero-gravity surface and the vacuum antigravity that controlled the outflow dynamics there.

In the present paper, we develop a systematic approach to the VLHF along the line traced in Paper I. We focus first on the physics which is responsible for the flow transition from the initial chaos to the present-day order. Two key aspects of the problem are the dynamical cooling of the expansion flow outside the local zero-gravity surface, which is studied analytically in Sect. 2, and the structure of the chaotic motions inside and outside this surface, which is the subject of computer simulations presented in Sect. 3. A discussion of the results is given and the major conclusions are summarized in Sect. 4.

\section{Vacuum cooling}

Following the considerations of Paper I, we adopt here that the VLHF takes its origin in the Little Bang developed inside the zero-gravity (ZG) surface around the Local Group. Each VLHF galaxy initially participates in the violent nonlinear dynamics of a many-body system of primeval galaxies and gaseous subgalactic clumps moving in the non-stationary gravitational potential of the early Local Group some $12-13$ Gyr ago. A population of dark matter subhalos might also occupy the group volume of $\simeq 1 \mathrm{Mpc}$ across (see Moore et al. 1999, and references therein). When a galaxy escapes from the group and crosses the ZG surface, it enters the area where the gravitational potential is nearly spherically symmetric and almost static. The former is clearly indicated by the shape of the local ZG surface that is nearly spherical and only slightly changes approaching in shape the perfect static sphere, asymptotically (Figs. 3-5, 6-8 below; see also Dolgachev et al. 2003, 2004 for more details). A typical trajectory of the small body (a dwarf galaxy) is nearly radial, in such a potential, as computer simulations show (Sect. 3). Therefore the first stage of the transition from chaos to order occurs within the
ZG surface, when an initially chaotic outflow of escaping galaxies becomes more or less radial when reaching the sphere. But this nearly radial flow remains rather "hot", and its radial velocity dispersion is still high at the surface. The outflow cooling is the second stage in the transition process.

Let us first discuss the cooling process. Due to the nearly radial symmetry of the outflow, a simple analytical treatment is possible for this second stage of the outflow evolution (the first stage is studied in detail later in Sect. 3). One may follow the motion of a small body (a test particle) outside the ZG surface along a radial trajectory, considering the particle as a "typical" member of the VLHF. Neglecting in the first approximation any (small) deviations of the "matter part" of gravitational potential from the spherical shape, we may describe the trajectory by the Newtonian equation of motion in the form

$\ddot{r}(t)=-G M / r^{2}+r / A_{\mathrm{V}}^{2}, \quad r \geq R_{\mathrm{V}}$.

The gravity of matter and the antigravity of vacuum are represented on the right-hand side of the equation by the terms of the opposite signs. Here $r$ is the distance of the body to the Local Group barycenter, $M=(1.5-2.5) \times 10^{12} M_{\odot}$ is the mass of the group, including both dark matter and baryons. The value $A_{\mathrm{V}}=\left(\frac{8 \pi G}{3} \rho_{\mathrm{V}}\right)^{-1 / 2} \simeq 1 \times 10^{28} \mathrm{~cm}$ is the vacuum's characteristic length (the Friedmann integral for vacuum - see Chernin 2001), and $\rho_{\mathrm{V}}=(7-8) \times 10^{-30} \mathrm{~g} \mathrm{~cm}^{-3}$ is the vacuum density. (Hereafter the speed of light $c=1$ in the formulas.)

It worth briefly explaining the structure of the second term on the right-hand-side of Eq. (1). This term comes from the wellknown General Relativity solution for a mass embedded in a vacuum: this is the Schwarzcschild-de Sitter static solution with the metric

$$
\mathrm{d} s^{2}=g_{00} \mathrm{~d} t^{2}-r^{2}\left(\sin \theta^{2} \mathrm{~d} \phi^{2}+\mathrm{d} \theta^{2}\right)-g_{11} \mathrm{~d} r^{2}
$$

where $g_{00}(r)=1 / g_{11}(r)=1-2 G M / r-\left(\frac{8 \pi G}{3} \rho_{\mathrm{V}}\right) r^{2}$. The radial coordinate $r$ is the distance from the central mass $M$. In the weak field limit (which is quite appropriate to the situation in the local volume), deviations from the Minkovski metric are small, and in the first approximation they are related - in accordance with a general rule - to the Newtonian potential $U(r)$ :

$g_{00}^{1 / 2} \simeq 1+U(r)$, so that $U(r)=-G M / r-\left(\frac{4 \pi G}{3} \rho_{\mathrm{V}}\right) r^{2}$.

This potential leads directly to the force (per unit mass) $-\frac{\mathrm{d} U}{\mathrm{~d} r}$ on the right-hand-side of Eq. (1). Some more details may be seen, for instance, in Chernin et al. (2006). To avoid any confusion, note that the antigravity force produced by vacuum is due to its energy density $\rho_{\mathrm{V}}$ and pressure $p_{\mathrm{V}}$ in the combination $\rho_{\mathrm{V}}+3 p_{\mathrm{V}}<$ 0 . This "effective gravitating density" is negative, which gives repulsion, not attraction. No force is due to the pressure gradient, because the vacuum pressure is perfectly uniform.)

The ZG surface, at which $\ddot{r}=0$, is a sphere of the radius

$R_{\mathrm{V}}=\left(G M A_{\mathrm{V}}^{2}\right)^{1 / 3} \simeq 1.3-1.7 \mathrm{Mpc}$

It is interesting that the mathematical structure of Eq. (1) is similar to the structure of the Friedmann cosmological equation for the global expansion factor $R(t)$ :

$\ddot{R}(t, \chi)=-G M(\chi) / R^{2}+R / A_{\mathrm{V}}^{2}$,

where $M(\chi)=$ const. $(t)$ is the mass of non-relativistic dark matter and baryons within the "co-moving" sphere of the Lagrangian radius $\chi$, and the Eulerian radius of a moving particle is $R(t, \chi)$. The similarity is not trivial because Eq. (1) comes 
from the static space-time, while Eq. (3) describes the spatial expansion of a non-stationary space-time. In the limit when $r$ and $R$ go to infinity, both space-times go to the same static de Sitter space-time, which is entirely controlled by the static and uniform vacuum. In this limit, both equations give the same velocitydistance law $\dot{r}=r / A_{\mathrm{V}}$ and $\dot{R}=R / A_{\mathrm{V}}$. Here the expansion rate, the "Hubble vacuum factor" $H_{\mathrm{V}}=1 / A_{\mathrm{V}}$ is a constant in both cases. This asymptotic property of the equations is significant, because it indicates that the motion of the particle we follow tends asymptotically to the cosmological regime. In the conventional units, $H_{\mathrm{V}}=1 / A_{\mathrm{V}} \simeq 60 \mathrm{~km} \mathrm{~s}^{-1} \mathrm{Mpc}^{-1}$, which is very near the observed value (see Sect. 1).

A close relation to cosmology is seen not only in the vacuum part of the Newtonian potential, but also in its matter part. Indeed, the total mass of the Local Group, together with the mass of all the bodies of the VLHF, are collected from the initially uniform cosmological matter distribution. An "unperturbed" cosmological volume containing the non-relativistic mass $M$ has the current radius

$R_{\mathrm{M}}=\left[M /\left(\frac{4 \pi}{3} \rho_{\mathrm{M}}\right)\right]^{1 / 3} \simeq 2 \mathrm{Mpc}$.

Here $\rho_{\mathrm{M}}=\rho_{\mathrm{D}}+\rho_{\mathrm{B}} \simeq 3 \times 10^{-30} \mathrm{~g} \mathrm{~cm}^{-1}$ is the sum of the dark matter mean density and the baryonic mean density at present. A near coincidence of the values $R_{\mathrm{M}}$ and $R_{\mathrm{V}}$ is obviously the result of the near coincidence of the mean dark matter density and the vacuum density in the current state of the Universe.

As one sees, the analytical model described by Eq. (1) has a direct connection to cosmology, since both its parameters, $M$ and $A_{\mathrm{V}}$, have a clear cosmological meaning: the cosmology parameters are imprinted on the dynamical background on which the local expansion flow develops.

The first integral of Eq. (1) expresses, as usual, the mechanical energy conservation for a particle moving in the potential $U$ :

$\frac{1}{2} \dot{r}^{2}=G M / r+\frac{1}{2}\left(r / A_{\mathrm{V}}\right)^{2}+E$,

where the total mechanical energy $E=$ const. is different, generally, for different particles. When the trajectory crosses the ZG surface and $r=R_{\mathrm{V}}$, one has from Eq. (5):

$\dot{r}^{2}=3\left(R_{\mathrm{V}} / A_{\mathrm{V}}\right)^{2}+2 E, \quad r=R_{\mathrm{V}}$.

It is easy to see that this is a minimal positive velocity of the outflow, as the velocity of the escaped particle increases at $r>R_{\mathrm{V}}$.

A "random" velocity $v$ may be introduced as a difference between the velocity $\dot{r}$ and a "regular" (asymptotic) velocity $r / A_{\mathrm{V}}$. Then the random velocity $v$ at $r=R_{\mathrm{V}}$, is

$v_{1}=\left(R_{\mathrm{V}} / A_{\mathrm{V}}\right)\left[\left(3+2 E A_{\mathrm{V}}^{2} / R_{\mathrm{V}}^{2}\right)^{1 / 2}-1\right]$.

As the trajectory reaches the distance $r$ outside the ZG surface, the random velocity $v(r)$ is given (for an arbitrary $E$ ) by the relation:

$v(r)=\frac{R_{\mathrm{V}}}{A_{\mathrm{V}}}\left[\left(\frac{r^{2}}{R_{\mathrm{V}}^{2}}-2+2 \frac{R_{\mathrm{V}}}{r}+2 \frac{v_{1} A_{\mathrm{V}}}{R_{\mathrm{V}}}+\frac{v_{1}^{2} A_{\mathrm{V}}^{2}}{R_{\mathrm{V}}^{2}}\right)^{1 / 2}-\frac{r}{R_{\mathrm{V}}}\right]$.

A comparison of $v(r)$ with $v_{1}$ enables us to find how the random velocity decreases along the particle trajectory. To do so, one may introduce the "vacuum cooling factor", $q_{\mathrm{V}} \equiv v_{1} / v$, which measures the efficiency of the random velocity suppression due to the vacuum:

$q_{\mathrm{V}}=\frac{v_{1} A_{\mathrm{V}}}{R_{\mathrm{V}}}\left[\left(\frac{r^{2}}{R_{\mathrm{V}}^{2}}-2+2 \frac{R_{\mathrm{V}}}{r}+2 \frac{v_{1} A_{\mathrm{V}}}{R_{\mathrm{V}}}+\frac{v_{1}^{2} A_{\mathrm{V}}^{2}}{R_{\mathrm{V}}^{2}}\right)^{1 / 2}-\frac{r}{R_{\mathrm{V}}}\right]^{-1}$
In the simplest case when the total energy is zero, $E=0$, the velocity $v_{1}=(\sqrt{3}-1) R_{\mathrm{V}} / A_{\mathrm{V}}$, and we have from Eq. (8):

$v(r)=\frac{R_{\mathrm{V}}}{A_{\mathrm{V}}}\left[\left(\frac{r^{2}}{R_{\mathrm{V}}^{2}}+2 \frac{R_{\mathrm{V}}}{r}\right)^{1 / 2}-\frac{r}{R_{\mathrm{V}}}\right]$.

Then the vacuum cooling factor in the parabolic expansion flow is

$q_{\mathrm{V}}(r)=\frac{v_{1}}{v(r)}=(\sqrt{3}-1)\left[\left(\frac{r^{2}}{R_{\mathrm{V}}^{2}}+2\right)^{1 / 2}-\frac{r}{R_{\mathrm{V}}}\right]^{-1}$.

One sees from Eqs. (7) and (11) that the random velocity is diminished by a factor $\simeq 3-$ from $\simeq 70$ to $\simeq 20 \mathrm{~km} \mathrm{~s}^{-1}-$ during the time when the body covers the path from $r=R_{\mathrm{V}}$ to $r \simeq 2 R_{\mathrm{V}} \simeq$ $3 \mathrm{Mpc}$. The resulting random velocity agrees with the observational value of the velocity dispersion (see Sect. 1). When the same body reaches, say, distances of 4 or $6 R_{\mathrm{V}}$, the cooling factor increases to $q_{\mathrm{V}}=12$ and $q_{\mathrm{V}}=56$, correspondingly.

If the random velocity $v(r)$ is small compared to the regular velocity $r / A_{\mathrm{V}}$ and $E=0$, one finds from Eq. (5) in the linear approximation:

$v \simeq R_{\mathrm{V}}^{3} /\left(A_{\mathrm{V}} r^{2}\right) \propto r^{-2}$.

Recall for comparison that the cosmological adiabatic cooling is described by the relation $v \propto R^{-1}$. Thus, the vacuum cooling of the local Hubble flow acts much more effectively than the adiabatic cooling does with the random motions on the expanding cosmological background. The second integral of Eq. (5) (for a parabolic orbit with $E=0$ ) has a well-known exact solution: $r \propto \sinh \left[\frac{3}{2} t / A_{\mathrm{V}}\right]^{2 / 3}$. Then the law of vacuum cooling in the linear approximation takes the form:

$q_{\mathrm{V}} \propto \sinh \left[\frac{3}{2} t / A_{\mathrm{V}}\right]^{4 / 3}$

One may - for another comparison - follow the dynamics of the same expansion flow, but in the absence of a cosmic vacuum. With the same statement of the problem as above, but for $\rho_{\mathrm{V}}=0$ and hyperbolic trajectories $(E>0)$, one finds an inertial asymptotic as $r$ goes to infinity: $\dot{r}=(2 E)^{1 / 2}$. This is a Hubbletype motion as well with the linear velocity-distance relation: $\dot{r}=r / t$, where the expansion rate, $H_{E}=1 / t$, depends on time. This suggests that the hyperbolic outflow may be cooled without vacuum (see also Sect. 3); but the cooling efficiency is remarkably lower in the absence of a vacuum. Indeed, taking $r / t$ as a regular motion velocity, one may introduce the random velocity $v$ by the relation $\dot{r}=r / t+v$. Then from Eq. (5) (without the vacuum term), one finds:

$v=(2 E)^{1 / 2}\left[\left(1+\frac{G M}{2 E r}\right)^{1 / 2}-1\right]$

In the linear approximation for $v /(2 E)^{1 / 2}<1$, Eq. (14) leads to the adiabatic relation $v \propto r^{-1}$. As a result, the cooling factor in the absence of vacuum, $q_{E} \propto r$, is significantly smaller than the vacuum cooling factor $q_{\mathrm{V}}$ of Eqs. (9), (11), (13).

\section{Computer simulations: from Little Bang to VLHF}

The VLHF evolution inside the local ZG surface cannot be described by any simple analytical model. It is controlled by complex nonlinear dynamics that may be followed - but only to some extent - with computer simulations. The Little Bang model 
Table 1. Ten simulation samples of Set I.

\begin{tabular}{lcccccccccc}
\hline \hline & 1 & 2 & 3 & 4 & 5 & 6 & 7 & 8 & 9 & 10 \\
\hline Reg. (now) & 86.30 & 98.85 & 69.41 & 145.20 & 91.95 & 123.08 & 104.24 & 100.82 & 115.05 & 74.91 \\
$\sigma_{\mathrm{V}}$ (now) & 30.29 & 31.46 & 32.53 & 27.16 & 36.81 & 31.29 & 20.93 & 29.52 & 36.80 & 34.66 \\
& & & & & & & & & & \\
Reg. (init) & -225.96 & -109.43 & -74.81 & 311.66 & -121.96 & 278.96 & -130.24 & -117.33 & -12.17 & -152.04 \\
$\sigma_{\mathrm{V}}$ (init) & 185.13 & 198.54 & 182.22 & 193.88 & 193.20 & 187.50 & 197.39 & 171.62 & 178.48 & 198.94 \\
\hline
\end{tabular}

(Byrd et al. 1994) and the picture of the early Local Group (van den Bergh 2003) provide important insights into this dynamics. Based on this, we developed a simplified approach in which the free fall of the two major galaxies of the Local Group on the cosmic vacuum background plays a central part. This is a "Little Bang Minimal Model" (LBMM), which takes into account that 1) the mass of the Local Group (LG) is mostly dark mass, and it strongly concentrates (Karachentsev et al. 2002b) to the two major galaxies of the group; 2) the dark matter halos of the Milky Way (MW) and one of the Andromeda Galaxy (M31) are nearly spherical; 3 ) the relative MW-M 31 motion is directed along the line of the centers of the galaxies, in accordance with the classic treatment (Kahn \& Woltjer 1959); 4) the LG is embedded in the cosmic vacuum, which is represented by a perfect medium with a uniform constant energy density.

In the LBMM, the gravity of the two major bodies of the Local Group and the antigravity of the vacuum are described by the Newtonian potential, which is similar to the potential of Sect. 2. The only difference is that now we have two gravitating bodies, not one. In the orthogonal barycenter coordinates $O x y$, in which $x$-axis is along the line of the MW and M 31 centers, the potential has the form

$U(x, y)=G\left(\frac{M_{1}}{r_{1}}+\frac{M_{2}}{r_{2}}+\frac{4 \pi}{3} \rho_{\mathrm{V}} r^{2}\right)$,

where

$r_{1}^{2}=\left(x-x_{1}\right)^{2}+y^{2}, \quad r_{2}^{2}=\left(x-x_{2}\right)^{2}+y^{2}$,

and $x_{1}$ and $x_{2}$ are the barycentric coordinates of the MW and M 31 centers, correspondingly. In the model below, we consider the motions of the VLHF galaxies as test particle motions in the axially symmetrical potential $U(x, y)$, so that the problem under consideration is reduced to the three-body restricted problem. The particle equation of motion is as follows:

$\frac{\mathrm{d}^{2} x}{\mathrm{~d} t^{2}}=\frac{\partial U}{\partial x}, \quad \frac{\mathrm{d}^{2} y}{\mathrm{~d} t^{2}}=\frac{\partial U}{\partial y}$

The computer integration of these equations gives the solution of the three-body restricted problem for any individual VLHF galaxy starting its motion within the Local Group. Despite its seemingly simple formulation, the test particle motion demonstrates a variety of dynamical patterns that might play, as we demonstrate below, the central part in the origin and evolution of the VLHF. The surface at which the radial force is zero is given by the condition that the scalar product of the acceleration vector and the radius-vector is zero; this leads to the equation

$x \frac{\mathrm{d}^{2} x}{\mathrm{~d} t^{2}}+y \frac{\mathrm{d}^{2} y}{\mathrm{~d} t^{2}}=0$.

The parameters of the model are the total mass of the MW $(0.6-1) \times 10^{12} M_{\odot}$, the total mass of M $31(0.9-1.5) \times 10^{12} M_{\odot}$, and the vacuum density $\rho_{\mathrm{V}}=(7-8) \times 10^{-30} \mathrm{~g} / \mathrm{cm}^{3}$. With the present separation $0.7 \mathrm{Mpc}$ and the present relative velocity $-120 \mathrm{~km} \mathrm{~s}^{-1}$, the two major galaxies of the LG started their motion toward each other 13.0-12.5 Gyr ago. At that moment in the past, the initial conditions are given for the particle motions. The sets of computer simulations were performed with a total of more than a thousand trajectories.

In Set I the masses of the two major galaxies are assumed to be 1 and 1.5 in the units of $10^{12} M_{\odot}$, and the vacuum density is assumed to be $7 \times 10^{-30} \mathrm{~g} / \mathrm{cm}^{3}$. The initial conditions for the particle trajectories are chosen in the moment $12.5 \mathrm{Gyr}$ ago in the three-dimensional axially-symmetrical phase space of positions and radial velocities of the LBMM. The phase space is scanned uniformly in the radial velocity range from -500 to $500 \mathrm{~km} \mathrm{~s}^{-1}$ and in the radial distance range from 0.15 to $0.80 \mathrm{Mpc}$. Both velocities and distances are related to the LG center-of-mass. In the simulations, 420 trajectories are integrated, and 397 of them are found in the VLHF distance interval (from 1 to $3 \mathrm{Mpc}$ ) at the present-day epoch. They form a bank of trajectories for the further statistical analysis.

To imitate the observed sample of the 22 VLHF galaxies (see their description in Paper I), we used samples of 22 trajectories each selected randomly from the bank of trajectories. Table 1 shows 10 of these simulation samples of Set I, each of which is characterized by the linear regression factor (calculated with the least-square method) and the corresponding linear velocity dispersion. They are both given for the present and the initial states of the sample. The present regression factor (the first line in Table 1) is the expansion time rate, which may be compared with the observed VLHF Hubble factor. The present velocity dispersion (the second line) is to be compared with the observed velocity dispersion. The initial regression factor (the third line) and the initial velocity dispersion (the fourth line) give a quantitative measure of the chaotic initial state of the flow.

Let us look, for instance, at the figures of Sample 1 in Table 1. The present state of the sample imitates the observed VLHF well. Indeed, the regression factor is $86 \mathrm{~km} \mathrm{~s}^{-1} \mathrm{Mpc}^{-1}$, which is within the two-sigma interval of the observed value of the local Hubble parameter (see Sect. 1). The flow is quiet and cool, and its velocity dispersion is $30 \mathrm{~km} \mathrm{~s}^{-1}$, which is also near the observed value. The current Hubble diagram for Sample 1 is shown in Fig. 1; it looks more or less similar to the real Hubble diagram for the VLHF (Karachentsev 2003b, 2005). The initial state of the sample differs drastically from the present one. The initial regression factor is negative for Sample 1 of Table 1. This means that the flow was contracting 12.5 Gyr ago, not expanding. The corresponding initial velocity dispersion is rather high, near $200 \mathrm{~km} \mathrm{~s}^{-1}$. Both figures show clearly that the initial state was highly chaotic and had nothing in common with an imaginary regular (or slightly disturbed) cosmological expansion flow on similar spatial scales some 12.5 Gyr ago.

Looking at the other samples of Table 1, we see that they are characterized to day by the regression factors that spread from 74 to $123 \mathrm{~km} \mathrm{~s}^{-1} \mathrm{Mpc}^{-1}$ and by the velocity dispersion within a rather narrow interval of $27-37 \mathrm{~km} \mathrm{~s}^{-1}$. Their initial states are 


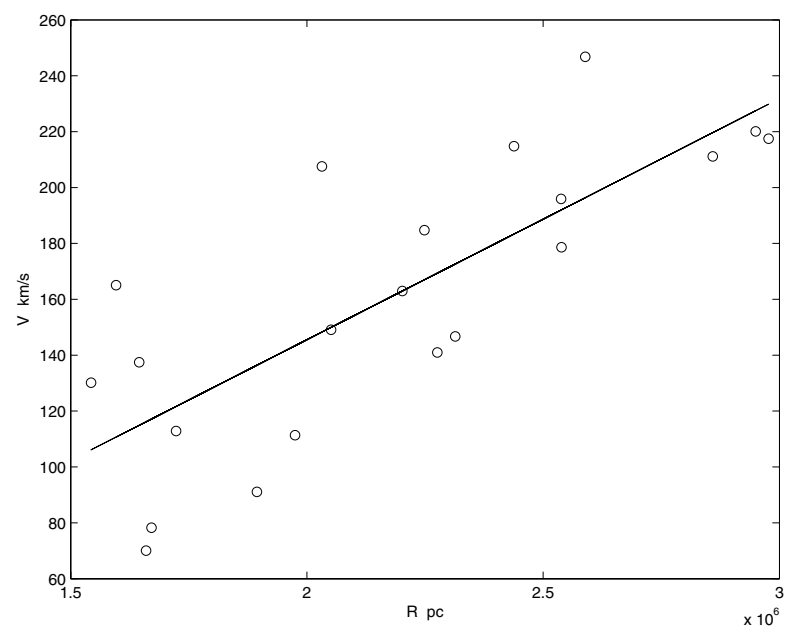

Fig. 1. Velocity-distance diagram for Sample 1 of Set I.
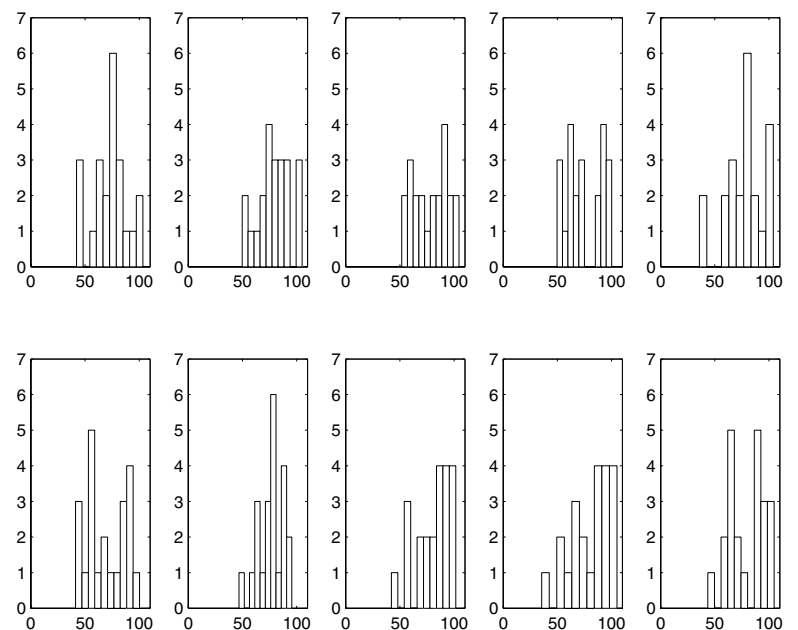

Fig. 2. Internal kinematic structure of simulation samples of Set I.

highly chaotic and may be both contracting or expanding. The initial velocity dispersion is near $200 \mathrm{~km} \mathrm{~s}^{-1}$ for all the samples. The internal dynamical structure of the samples of Table 1 in the present-day state is illustrated in Fig. 2, with the distributions of the "individual expansion rates", which are velocity-distance ratios for the members of a sample. We can see that, in a typical case, there is a dominant group of trajectories (mostly hyperbolical ones) that determines the overall statistical characteristics of the samples.

Taking Sample 1 of Table 1 as a typical one, we show its 22 trajectories in the plane of symmetry of the Local Group - see Fig. 3. The local ZG surface is shown in the section of this plane; both initial and present day shapes of the surface are given in the figure. These the surface is rather circular and changes with time rather slightly for the last $12.5 \mathrm{Gyr}$. Most of the trajectories intersect the ZG surface along nearly radial directions. The geometry of most of the trajectories is rather simple: they are very close to straight lines, outside the ZG surface (taken into account in the discussion of Sect. 2). At least in part, this geometry is due to the initial conditions at which the velocities only had a radial component.

A possible effect of initial non-radial velocities was especially studied for the same sample 1. For this goal, the initial LBMM phase space was extended to include transverse velocities. Figure 4 shows the trajectories of the same galaxies

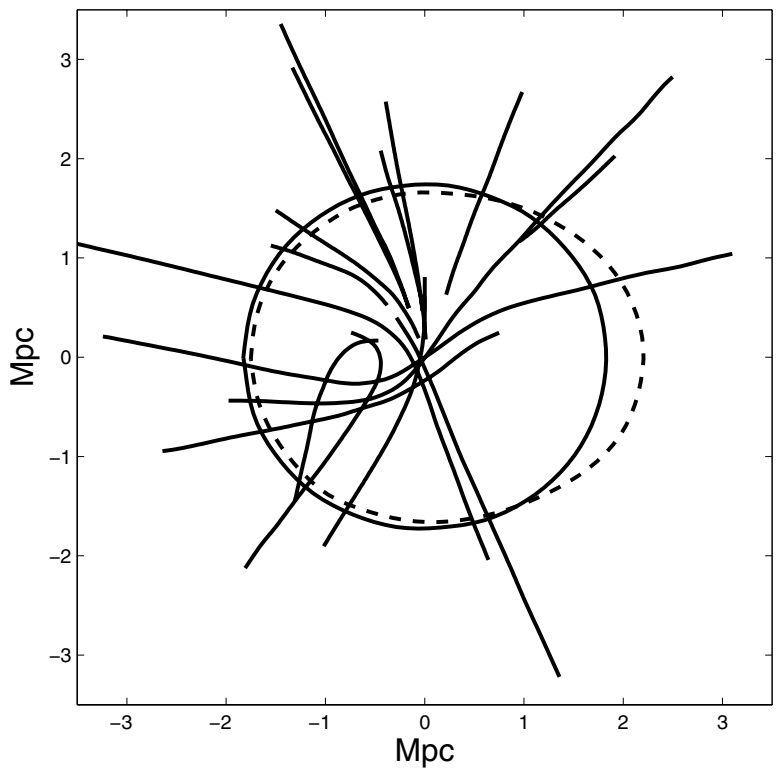

Fig. 3. Trajectories of Sample 1 of Set I. The initial "egg" shape of the ZG surface becomes closer to a sphere, as the two galaxies approach each other with time.

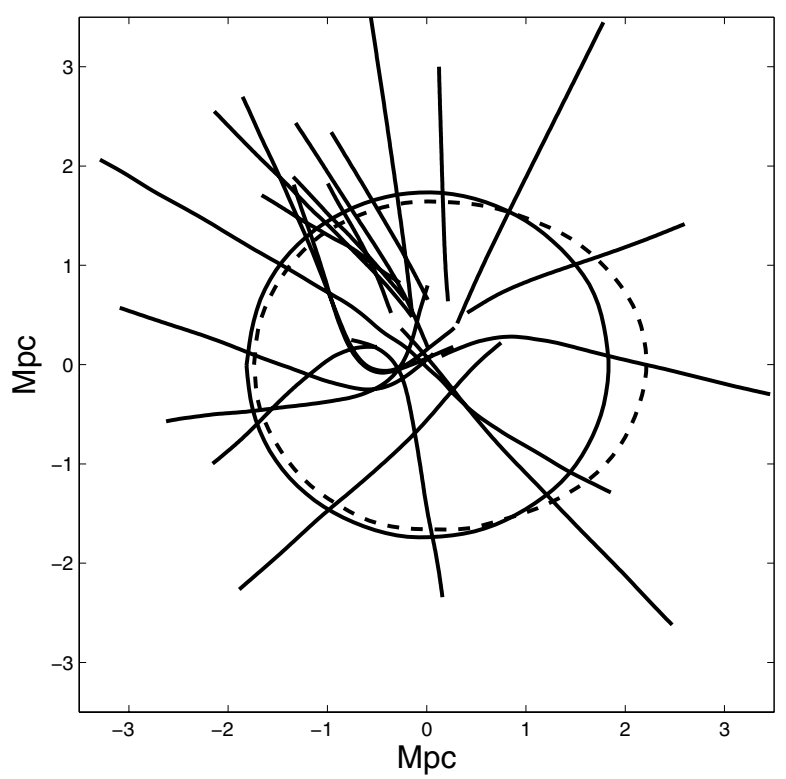

Fig. 4. Same for additional $\pm 50 \mathrm{~km} \mathrm{~s}^{-1}$ initial transverse velocities.

as in Fig. 3, but with the additional transverse velocity, which is initially $\pm 50 \mathrm{~km} \mathrm{~s}^{-1}$ for the members of the sample. Figure 5 illustrates the effect of initial transverse velocities of $\pm 100 \mathrm{~km} \mathrm{~s}^{-1}$. Finally, Fig. 6 shows (not realistic) trajectories with zero initial radial velocity and with initial transverse velocities of $\pm 100 \mathrm{~km} \mathrm{~s}^{-1}$. This is actually a case of an initial, rapid rotation of the masses of the ensemble around the LG barycenter, and most of the trajectories prove to stay within the ZG surface. With the exception of the unrealistic example of Fig. 6, the initial transverse velocities do not significantly alter the simple nearly straight-line geometry of typical trajectories outside the $\mathrm{ZG}$ surface. Table 2 gives quantitative characteristics of the effect of the transverse velocities: the present expansion rate (the first line) and velocity dispersion. A comparison with the data for Sample 1 of Table 1 shows that the realistic initial transverse velocities (Cols. 2, 3) are compatible with the major trend in 


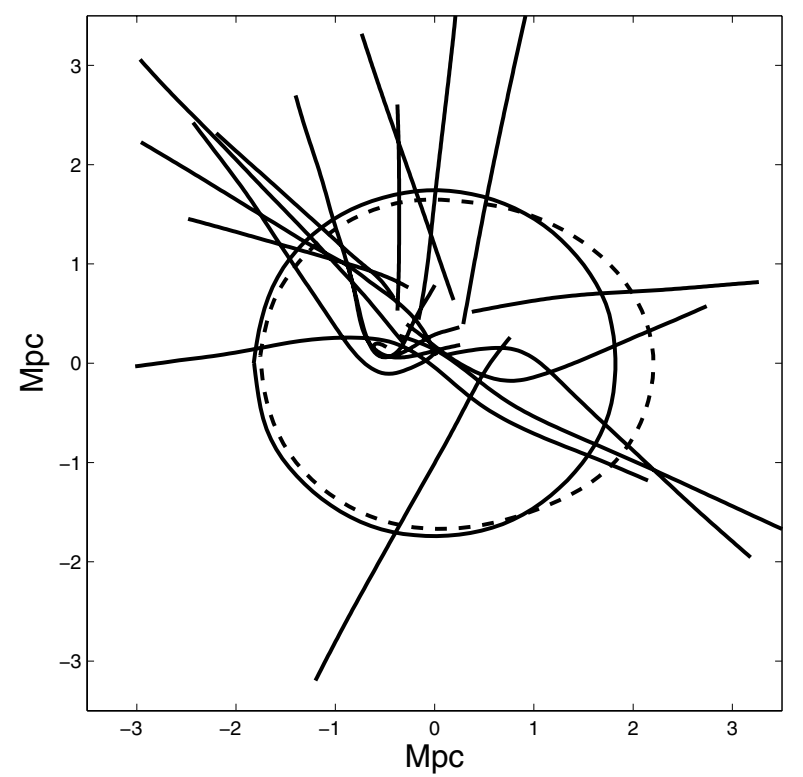

Fig. 5. Same for additional $\pm 100 \mathrm{~km} \mathrm{~s}^{-1}$ initial transverse velocities.

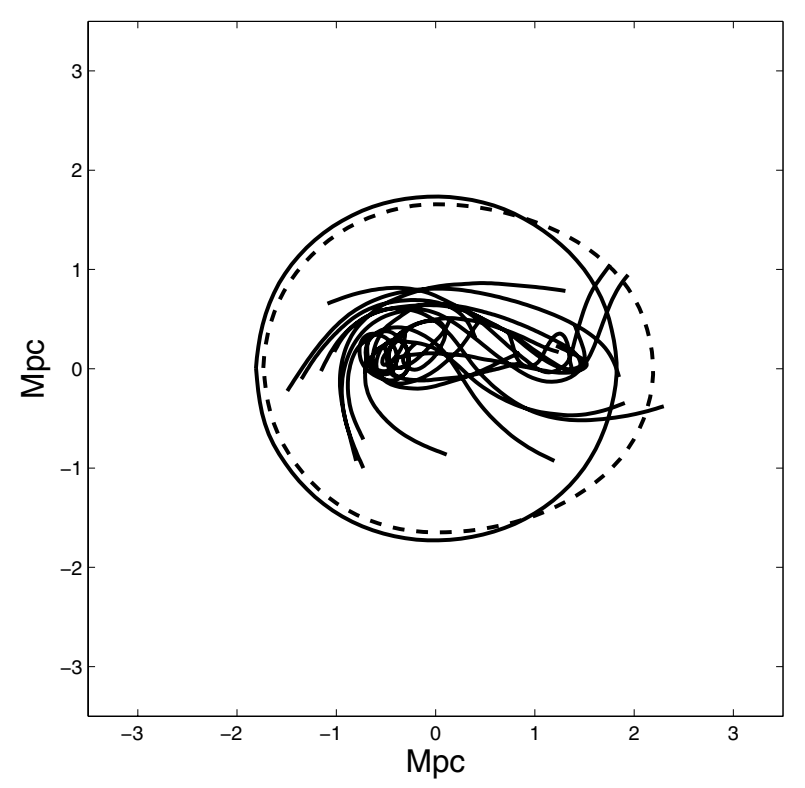

Fig. 6. Same for $\pm 100 \mathrm{~km} \mathrm{~s}^{-1}$ initial transverse velocities and no radial velocities.

Table 2. The effect of the transverse velocities for Sample 1 of Set I.

\begin{tabular}{lcccc}
\hline \hline & 1 & $V r \pm 50$ & $V r \pm 100$ & $0 \pm 100$ \\
\hline Reg. (now) & 86.30 & 84.47 & 78.02 & 27.56 \\
$\sigma_{\mathrm{V}}$ (now) & 30.29 & 28.45 & 24.60 & 65.27 \\
\hline
\end{tabular}

the sample evolution. They do not change the statistical characteristics of the outflow present-day state significantly. Indeed, with the transverse velocities \pm 50 and $\pm 100 \mathrm{~km} \mathrm{~s}^{-1}$, the expansion rate decreases slightly (from 86 to 85 and $78 \mathrm{~km} \mathrm{~s}^{-1}$, correspondingly) keeping within the same two-sigma interval of the observation figure. The velocity dispersion does not change significantly either. Only in the unrealistic case of zero initial radial velocities (Col. 4) do the figures turn out to be considerably different.

In Set II, the same parameters of integration and initial conditions as in Set I were used in a model without a vacuum. The results are given in Table 3, which may be compared with Table 1 of Set I. One can see that the major trend in the dynamical evolution is the same: this is a transformation of a flow from chaos to order. The similarity with the vacuum model in this respect is expected, in view of the analysis in Sect. 2. Indeed, the existence of regular asymptotic states with the linear velocity-distance relation is fairly universal for hyperbolic trajectories, as is seen from the analytical treatment in Sect. 2 . The vacuum antigravity effect enhances the trend to order essentially, and this is revealed more clearly at larger distances in the local expansion flows. With the modest distances of the VLHF, the quantitative difference between the two models is not too significant. One only notices somewhat lower values of the final regression factor, between 47 and $105 \mathrm{~km} \mathrm{~s}^{-1} \mathrm{Mpc}^{-1}$, and somewhat higher values of the velocity dispersion in a narrow range from 32 to $38 \mathrm{~km} \mathrm{~s}^{-1}$, in the models without vacuum.

Finally, in Set III, lower (than in Sets I, II) masses of the two major galaxies of the Local Group are used, which are 0.6 and 0.9 in the units of $10^{12} M_{\odot}$, and a higher vacuum density: $8 \times 10^{-30} \mathrm{~g} \mathrm{~cm}^{-3}$. The initial conditions for the particle trajectories are chosen in the moment $13 \mathrm{Gyr}$ ago (the moment when the MW and M31 start their motion towards each other), and the phase space is scanned in a narrower radial velocity range, from -150 to $+150 \mathrm{~km} \mathrm{~s}^{-1}$, to decrease the number of hyperbolic trajectories. The radial distances range from 0.15 to $0.80 \mathrm{Mpc}$, as in Set I. After integration, 327 trajectories are found in the distance range 1-3 Mpc; 47 of them show too close encounters with the MW or M31 (at less than $50 \mathrm{kpc}$ distance from their centers), and they are not included in the bank of trajectories for the statistical analysis. The results of the analysis of Set III are given in Tables 4 and 5 and Figs. 7-10.

The regression factor (the first line of Table 4) is somewhat lower than in Sets I and II: in the range from 44 to $70 \mathrm{~km} \mathrm{~s}^{-1} \mathrm{Mpc}^{-1}$. It is compatible with the observation value, but closer now to the characteristic vacuum value $H_{\mathrm{V}} \simeq$ $60 \mathrm{~km} \mathrm{~s}^{-1} \mathrm{Mpc}^{-1}$. This is clearly because of a relatively stronger vacuum effect for the lower matter masses and higher vacuum density in Set III. Because of the same physical cause, the vacuum cooling is more effective in Set III, so that the velocity dispersion is noticeably lower now ranging from 15 to $25 \mathrm{~km} \mathrm{~s}^{-1}$. Meanwhile the initial states of the model flows are very chaotic with the velocity dispersion higher than $100 \mathrm{~km} \mathrm{~s}^{-1}$ (the fourth line of Table 4). The initial and final values of the dispersion correspond to a rather large vacuum cooling factor $q_{\mathrm{V}} \simeq 4-6$ (see for comparison Sect. 2). Table 5 illustrates (as in Table 2) a weak effect of transverse initial velocities in Set III.

Thus, the results of integration of more than a thousand trajectories of the LBMM dwarf galaxies enable us to simulate the origin of the VLHF and its dynamical evolution both within the zero-gravity surface and outside it. In agreement with the analytic considerations of Sect. 2, the simulations definitely prove the real possibility of the VLHF evolution from the initial Little Bang chaos some $12-13$ Gyr ago to the order and regularity at present.

\section{Discussion and conclusions}

The recent high-precision mapping of the local velocity field and high-accuracy distance measurements in the Local Volume (Karachentsev et al. 2000, 2001, 2002a, 2002b, 2003b, 2004, 2005; Karachentsev \& Makarov 2000; Ekholm et al. 2001; Davidge \& van den Bergh 2001; Teerirorpi \& Paturel 2002; Thim et al. 2003; Paturel \& Teerikorpi 2004; Reindl et al. 2005) have given a clear picture of the nearest Universe. Important 
Table 3. Ten simulation samples of Set II.

\begin{tabular}{|c|c|c|c|c|c|c|c|c|c|c|}
\hline & 1 & 2 & 3 & 4 & 5 & 6 & 7 & 8 & 9 & 10 \\
\hline Reg. (now) & 74.93 & 60.55 & 86.66 & 85.87 & 105.07 & 97.17 & 87.03 & 56.57 & 78.80 & 46.80 \\
\hline$\sigma_{\mathrm{V}}($ now $)$ & 36.99 & 37.50 & 29.59 & 39.59 & 37.15 & 32.15 & 34.98 & 36.91 & 34.22 & 33.49 \\
\hline Reg. (init) & -19.39 & 153.65 & 123.25 & 301.90 & 362.22 & -3.34 & -173.21 & -25.99 & -331.72 & 80.69 \\
\hline$\sigma_{\mathrm{V}}$ (init) & 198.97 & 185.39 & 198.75 & 192.67 & 174.79 & 203.86 & 209.54 & 212.12 & 201.32 & 203.77 \\
\hline
\end{tabular}

Table 4. Ten simulation samples of Set III.

\begin{tabular}{lcccccccccc}
\hline \hline & 1 & 2 & 3 & 4 & 5 & 6 & 7 & 8 & 9 & 10 \\
\hline Reg. (now) & 63.48 & 70.10 & 48.41 & 52.34 & 68.54 & 67.22 & 61.95 & 59.41 & 60.10 & 43.76 \\
$\sigma_{\mathrm{V}}$ (now) & 21.60 & 23.65 & 21.68 & 21.91 & 14.69 & 24.65 & 22.22 & 17.99 & 23.49 & 20.25 \\
Reg. (init) & 213.91 & 18.02 & 2.29 & -175.15 & 53.39 & -26.97 & 179.06 & -110.64 & -72.55 & -152.17 \\
$\sigma_{\mathrm{V}}$ (init) & 109.92 & 109.90 & 128.54 & 115.62 & 121.99 & 117.23 & 104.48 & 113.56 & 118.21 & 108.26 \\
\hline
\end{tabular}

Table 5. The effect of the transverse velocities for Sample 1 of Set III.

\begin{tabular}{lcccc}
\hline \hline & 1 & $V r \pm 50$ & $V r \pm 100$ & $0 \pm 100$ \\
\hline Reg. (now) & 63.48 & 54.92 & 51.67 & 18.32 \\
$\sigma_{\mathrm{V}}$ (now) & 21.60 & 19.22 & 14.44 & 87.94 \\
\hline
\end{tabular}

observed features of the local expansion flow are discussed by Whiting (2003) and by Axenides \& Perivolaropoulos (2002).

The data have reliably confirmed the earlier conclusions by Sandage et al. (1972) and Sandage $(1986,1999)$ that there is a regular local Hubble flow of expansion deep inside the cell of matter uniformity. It has the expansion rate near the global Hubble constant and the remarkably low velocity dispersion. An understanding of the physical nature of the local Hubble flow has come with the discovery of cosmic vacuum (or the cosmological constant). This omnipresent vacuum with its perfectly uniform dominant density provides the dynamical conditions for a regular flow in the highly non-uniform matter distribution (Chernin et al. 2000; Chernin 2001; Baryshev et al. 2001; Karachentsev et al. 2003a; Thim et al. 2003; Teerikorpi et al. 2005; Sandage et al. 2006; Chernin et al. 2006).

In Paper I, we started detailed studies along this line focusing on the very local $(\leq 3 \mathrm{Mpc})$ area of the flow where it starts. An assumption was made that the very local Hubble flow was generated in the Little Bang due to the chaotic dynamics of the forming Local Group. In the present paper, we study the physics of the transition of the flow from the Little Bang initial chaotic state to its presently observed quasi-regular state.

The transition from chaos to order is known in various areas of the natural (and also social) sciences, but is poorly understood still. It has been found in fundamental nonlinear physics and, in particular in the theory of turbulence, that a high degree of complexity is a characteristic of this process. Remarkably enough, in the case of the very local Hubble flow (VLHF), the nonlinear phenomena we face may be clarified and treated in a fairly simple way. The LBMM enables us to describe the problem in an explicit form and find both qualitative and quantitative solutions to it. The solution we obtain confirms the major approach of Paper I.

Indeed, the picture of the VLHF initial chaos is not too puzzling in our model. It is, first, due to the random choice of the initial states of the VLHF members in the phase space of the Local Group embedded in the cosmic vacuum. Second, it is due to the nonlinear three-body dynamics of gravitational scattering of a test mass on a non-stationary massive binary system. In the gravitational scattering, the dynamical instability is the major

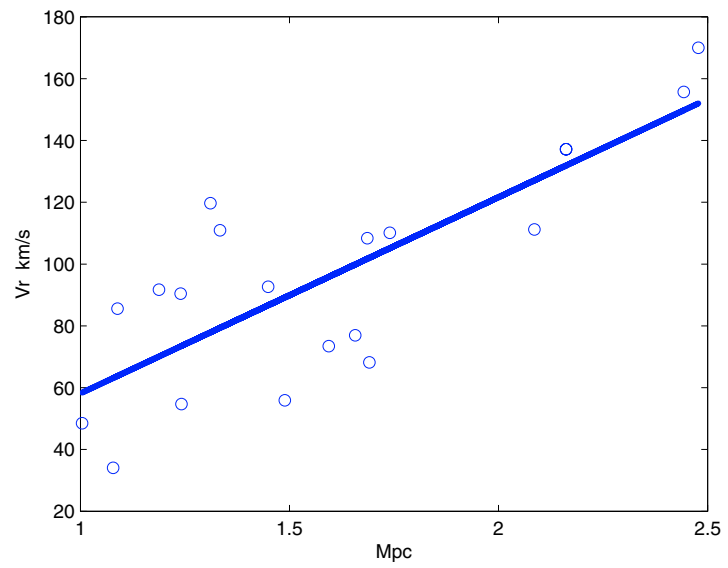

Fig. 7. Velocity-distance diagram for Sample 1 of Set III.

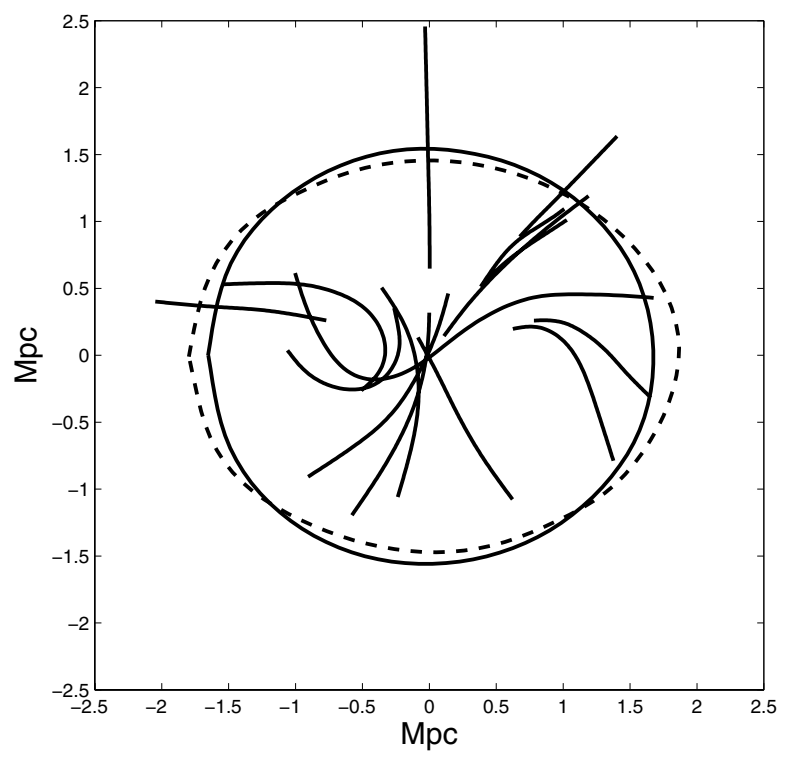

Fig. 8. Trajectories of Sample 1 of Set III.

physical factor that controls it and that introduces the Poincarétype chaos to the system (see reviews by Valtonen \& Mikkola 1991; Chernin \& Valtonen 1998). Numerical solutions and computer simulations of this process can be performed easily. Under the particular conditions of the LBMM, the simulations may be reduced to the standard integration of the three-body restricted 


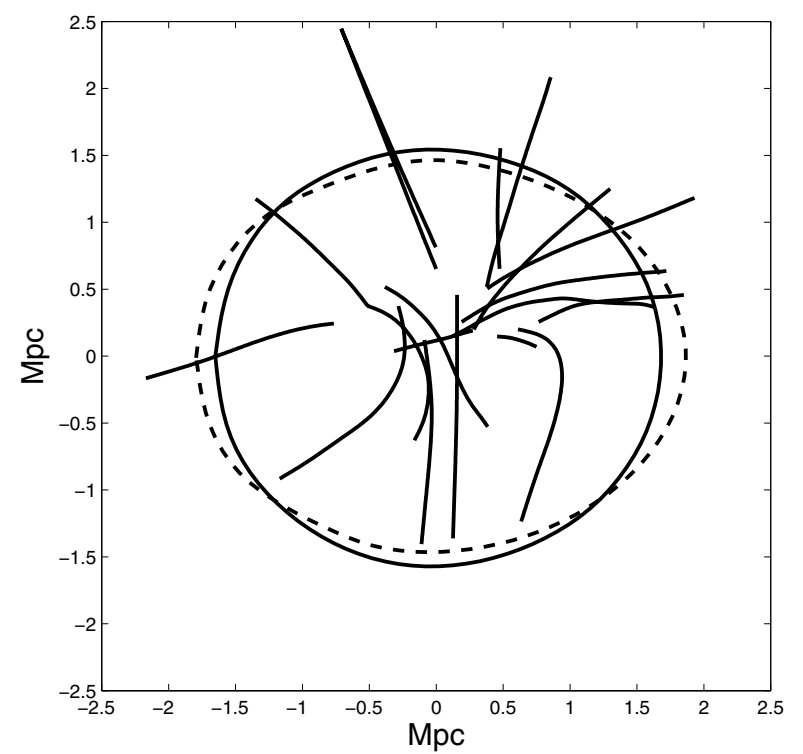

Fig. 9. Same for additional $\pm 50 \mathrm{~km} \mathrm{~s}^{-1}$ initial transverse velocities.

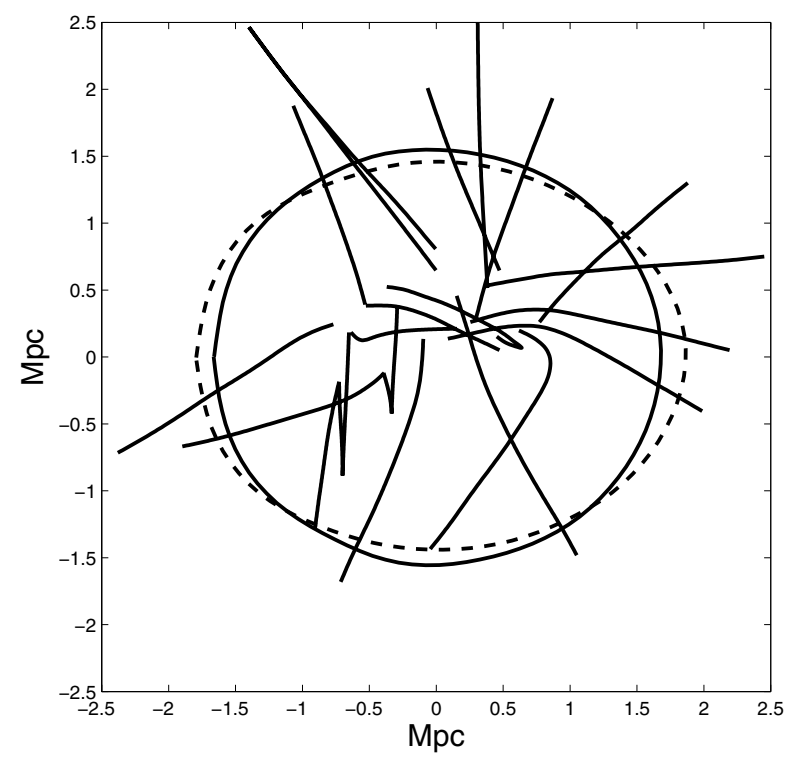

Fig. 10. Same for additional $\pm 100 \mathrm{~km} \mathrm{~s}^{-1}$ initial transverse velocities.

problem (Sect. 3). A new feature is involved in our simulations, the vacuum background on which the process develops.

It turns out that, when a test body escapes from the twobody gravitational potential, the dynamical instability gradually ceases with the distance from the system barycenter, and the third body occurs in the potential that becomes more and more centrally symmetrical. This is clearly seen from the computed geometry of the local zero-gravity (ZG) surface. In fact, the escaped body is affected mainly by a simple, nearly centrallysymmetrical potential outside this surface. This potential is practically static: its evolution is very slow, as seen from the comparison of the initial and final shape and the size of the zero-gravity surface (Figs. 3-6, 8-10). Under these conditions, the motion of the scattered body becomes more and more regular. In a sample of bodies imitating the VLHF, it leads to a picture of a flow that acquires the linear velocity-distance relation, and the deviations from this relation decrease with time.

A cosmic vacuum that takes over outside the ZG surface of the Local Group introduces additional stability and regularity into the sample evolution. First, it produces a perfectly symmetrical and static major contribution to the gravitational potential. Second, it accelerates the escaped bodies and so leads to larger distances from the system barycenter for the lifetime of the system. The computer simulations show that, even at small distances, the evolutionary trend introduced by the cosmic vacuum is quite recognizable, while it may not be too quantitatively significant for highly hyperbolic trajectories (see Tables 1 and 3). Since parabolic and elliptical trajectories are most common in the real VLHF (see more in Chernin et al. 2006), the dynamical effect of a vacuum is actually more prominent and leads to the formation of the expansion rate, which is very near the characteristic vacuum value (Table 4). Another quantitative measure of the efficiency of this evolution is the "vacuum cooling factor" introduced in Sect. 2. Both analytical considerations and computer simulations demonstrate that the cosmic vacuum provides the most effective mechanism of the flow transition from chaos to order, especially for non-hyperbolic trajectories: it decrease the initial velocity dispersion in the local Hubble flow considerably (5-6 times - see Table 4). It is the cosmic vacuum cooling that makes the whole flow of expansion into a self-consistent regular cosmological phenomenon to its entire extent inside the cosmic cell of uniformity.

The results presented above are in general agreement with the large N-body $\Lambda$ CDM cosmological simulations (Ostriker \& Suto 1990; Suto et al. 1992; Nagamine et al. 1999, 2000; Macò et al. 2005) oriented on relatively small scales. These simulations give important insights into the early history of the local Universe, when the matter of its volume started to separate from the general cosmological expansion at the cosmic age of 0.5-1.5 Gyr. The results complement ours and demonstrate that the local Universe, with a massive group in its center and the cool expansion outflow from the center, is rather typical for relatively small scales. Such local structures emerge in a natural way from the standard picture of gravitational instability with the Harrison-Zeldovich spectrum. These simulations have shown as well that the dark energy is necessary for explaining cool local flows. A theoretical treatment by Leong \& Saslaw (2004) within the framework of many-body gravitational clustering also shows that the observed small departures from the regular Hubble flow beyond the Local Group are highly probable. They also find that low mass groups, like the Local Group, dominate in the clustering process, and the Hubble flow is not significantly disturbed around them.

A full N-body cosmological approach shows in addition that the dynamics of the initial Little Bang might be much richer than one described by the LBMM. In particular, about 1000 dark matter subhalos may be expected within $700 \mathrm{kpc}$ from the center of a group like the Local Group (Klypin et al. 1999; Moore et al. 1999; Gao et al. 2003; Diemand et al. 2003). The dynamical friction and the tidal interactions produced by the subhalo population may modify individual trajectories of test galaxies quite substantially. It is interesting to follow more closely other collective processes that may develop in the forming group. In this way, one can clarify their effect on the escape rate from the system and the initial conditions for the outflow galaxies. These aspects of the dynamics of evolving groups will be the subject of a special detailed study in Paper III of this series.

Acknowledgements. We thank G. Byrd, A. Cherepashchuk, Yu. Efremov, A. Silbergleit, P. Teerikorpi and A. Zasov for discussions. DM acknowledges support from INTAS grant 03-55-1754. The work is also partly supported by the RFBR grants 06-02-16366 and 07-02-00005 and joint DFG-RFBR grant 06-02-04017. 


\section{References}

Baryshev, Yu. V., Chernin, A. D., \& Teerikorpi, P. 2001, A\&A, 378, 729 Byrd, G., Valtonen, M., McCall, M., \& Innanen, K. 1994, AJ, 107, 2055 Chernin, A. D. 2001, Physics-Uspekhi, 44, 1099

Chernin, A. D., \& Valtonen, M. J. 1998, New AR, 42, 41

Chernin, A. D., Teerikorpi, P., \& Baryshev, Yu. V. 2003, Adv. Space Res., 31, 459

Chernin, A. D., Karachentsev, I. D., Valtonen, M. J., et al. 2004, A\&A, 415, 19 (Paper I)

Chernin, A. D., Teerikorpi, P., \& Baryshev, Yu. V. 2006, A\&A, 456, 13

Diemand, J., Moore, B., \& Stadel, J. 2004, MNRAS, 352, 535

Dolgachev, V. P., Domozhilova, L. M., \& Chernin, A. D. 2003, Astr. Rep., 47, 728

Dolgachev, V. P., Domozhilova, L. M., \& Chernin, A. D. 2004, Astr. Rep., 48, 787

Gao, L., White, S. D. M., Jenkins, A., Stoehr, F., \& Springel, V. 2004, MNRAS, 355,819

Giovanelli, R., Dale, D., Haynes, M., Hardy, E., \& Campusano, L. 1999, ApJ, 525,25

Kahn, F. D., \& Woltjer, L. 1959, ApJ, 130, 705

Karachentsev, I. D. 2005, AJ, 129, 178

Karachentsev, I. D., Sharina, M. E., Grebel, E. K., et al. 2000, ApJ, 542, 128

Karachentsev, I. D., Sharina, M. E., Dolphin, A. E., et al. 2002a, A\&A, 385, 21
Karachentsev, I. D., Sharina, M. E., Makarov, D. I., et al. 2002b, A\&A, 389, 812 Karachentsev, I. D., Chernin, A. D., \& Teerikorpi, P. 2003a, Astrophysics, 46, 399

Karachentsev, I. D., Makarov, D. I., Sharina, M. E., et al. 2003b, A\&A, 398, 479 Karachentsev, I. D., Dolphin, A. E., Tully, R. B., et al. 2006, AJ, 131, 1361

Klypin, A., Gottlober, S., Kravtsov, A. V., \& Khokhlov, A. M. 1999, ApJ, 516, 530

Leong, B., \& Saslaw, W. C. 2004, ApJ, 608, 636

Macciò, A. V., Governato, F., \& Horellow, C. 2005, MNRAS, 359, 941

Moore, B., Quinn, T., Governato, F., Stadel, J., \& Lake, G. 1999, MNRAS, 310, 1147

Perlmutter, S., Aldering, G., Goldhaber, G., et al. 1999, ApJ, 517, 565

Reindl, B., Tammann, G. A., Sandage, A., \& Saha, A. 2005, ApJ, 624, 532

Riess, A. G., Filippenko, A. V., Challis, P., et al. 1998, AJ, 116, 1009

Sandage, A. 1986, ApJ, 307, 1

Sandage, A. 1999, ApJ, 527, 479

Sandage, A., Tammann, G., \& Hardy, E. 1972, ApJ, 172, 253

Sandage, A., Tammann, G., Saha, A., et al. 2006, ApJ, 653, 843

Teerikorpi, P. 1997, ARA\&A, 35, 101

Teerikorpi, P., Chernin, A. D., \& Baryshev, Yu. V. 2005, A\&A, 440, 791

Thim, F., Tammann, G., Saha, A., et al. 2003, ApJ, 590, 256

Valtonen, M. J., \& Mikkola, S. 1991, ARA\&A, 29, 9

van den Bergh, S. 2003 [arXiv: astro-ph/0305042]

Whiting, A. B. 2003, ApJ, 587, 186 